Ann. Zootech., I975, 24 (3), 363-37I.

\title{
INFLUENCE DE LA MACHINE DE RÉCOLTE SUR LES QUANTITÉS D'ENSILAGE INGÉRÉES ET LES PERFORMANCES DES VACHES LAITIERES
}

\author{
J. P. DULPHY et C. DEMARQUILLY \\ avec la collaboration technique de J.-P. Axprizu \\ Laboratoire des Aliments, \\ Centre de Recherches de Clermont-Ferrand, I. N.R. A., \\ Theix, Saint Genès Champanelle, 63110 Beaumont
}

\section{RÉSUMÉ}

Nous avons comparé sur des vaches laitières trois ensilages de fléole récoltés sur la même prairie avec trois machines différentes : ensileuse à tambour-hacheur (brins courts : en moyenne $0,4 \mathrm{~cm}$ ), ensileuse à double-coupe (brins moyens : $8,5 \mathrm{~cm}$ ) et ensileuse à fléaux (brins longs : Io $\mathrm{cm}$ ). Les trois ensilages préparés avec addition d'acide formique lors de la récolte étaient très bien conservés.

Ces ensilages ont été distribués à volonté sans foin à 3 lots comparables de vaches laitières. Quel que soit l'ensilage reçu les vaches ont reçu en complément un aliment concentré à raison de $0,38 \mathrm{UF}$ par $\mathrm{kg}$ de lait 4 p. Ioo produit au-dessus de I I $\mathrm{kg}$ par jour. La période expérimentale s'est déroulée du $73^{\mathrm{e}}$ au $143^{\mathrm{e}}$ jour de lactation en moyenne.

Les trois ensilages ont été ingérés en quantités significativement différentes : respectivement $2,24, \mathrm{I}, 83$ et $2,00 \mathrm{~kg}$ pour les ensilages à brins courts, moyens et longs. La production de lait à 4 p. roo de matières grasses a été significativement supérieure avec l'ensilage à brins courts : respectivement $\mathrm{I} 8,5$ contre $\mathrm{I} 6,7$ et $\mathrm{I}_{7, \mathrm{I}} \mathrm{kg}$ essentiellement à cause du taux butyreux lui-même significativement plus élevé : $4^{\circ}, \mathrm{I}$ contre 37,3 et $36,3 \mathrm{p}$. Ioo. La teneur en matières azotées du lait a également été plus élevée avec l'ensilage à brins courts: 35 , I contre $3 \mathrm{r}, \mathrm{o}$ et 32, $\mathrm{p}$. I 000 .

Les variations de poids pendant la période expérimentale ont été respectivement de $+2 \mathrm{I} 3 \mathrm{~g}$, - I 52 et - $3 \mathrm{~g} / \mathrm{jour}$.

Le hachage fin des ensilages a donc permis une augmentation notable des performances des vaches laitières. Ce résultat a été obtenu non seulement par une augmentation des quantités ingérées, mais aussi, semble-t-il, par le fait que les ensilages à brins courts ont eu une valeur énergétique nette plus élevée que celle des ensilages à brins moyens ou longs $(+0,05 \mathrm{UF} / \mathrm{kg}$ de matière sèche). La couverture des besoins énergétiques par la ration de base, en plus des besoins d'entretien, a été de $13,7 \mathrm{~kg}$ de lait 4 p. Ioo pour l'ensilage à brins courts contre seulement $8,5 \mathrm{~kg}$ en moyenne pour les deux autres ensilages. 


\section{INTRODUCTION}

Les vaches laitières ingèrent les ensilages d'herbe en coupe directe en quantités relativement faibles, généralement comprises entre $I, 5$ et $\mathrm{I}, 8 \mathrm{~kg}$ de matière sèche pour roo $\mathrm{kg}$ de poids vif (cf. PREsthegGe, I959, CAstle et WAtson, I970 $a$ et $b$ ). Aussi le préfanage a-t-il souvent été préconisé pour augmenter les quantités d'ensilage ingérées et diminuer les quantités d'aliments concentrés à distribuer.

Nous avons, quant à nous, montré que le hachage fin du fourrage lors de la récolte des ensilages permettait d'augmenter de façon importante les quantités ingérées par les moutons (DUlPhy et I)EMARQuilly, 1973) et dans une moindre mesure par les génisses de I an (DulPhy et DeMARQUIL,Y, I975).

Il existe très peu d'études à ce sujet sur les vaches laitières. Gordon et al. (I958) ont comparé des fourrages ensilés longs ou hachés. Le hachage a augmenté les quantités ingérées ( - II,4 p. Ioo) et la production laitière dans le premier essai, mais pas dans le second dans lequel les deux ensilages comparés étaient très mal conservés. Dans deux essais réalisés par MuRDOCH (I965) le hachage a augmenté les quantités d'ensilages ingérées $(-+$ Io à +20 p. Ioo) par des vaches laitières taries.

Aussi avons-nous comparé sur des vaches laitières trois ensilages de graminées correspondant à trois finesses de hachage différentes : brins courts (ensileuse à tambourhacheur), brins moyens (ensileuse à double coupe), brins longs (ensileuse à fléaux).

\section{MATÉRIEI、 ET MÉTHODES}

\section{Fourrages}

La même prairie de fléole à Orcival ( quelques jours avant le début de l'épiaison du premier cycle de végétation avec trois machines différentes :

- une ensileuse à couteaux sur tambour, hachant lc fourrage en brins courts (longueur moyenne mesurée de $0,4 \mathrm{~cm})$;

- une ensileuse à double coupe (fléaux en I et plateau hacheur) hachant le fourrage en brins moyens (en moyenne $8.5 \mathrm{~cm}$ ) ;

- une ensileuse à fléaux (fléaux en cuiller) hachant le fourrage en brins longs (en moyenne IO $\mathrm{cm}$ ).

Nous appellerons les cnsilages correspondants, des ensilages à brins courts, moyens et longs. On notera cependant que l'ensilage à brins dits "moyens $n$ a, contrairement à ce que nous espérions, une longucur de brins très peu inférieure à celle de l'ensilage à brins dits "longs".

Ces trois ensilages ont été récoltés respectivement les 27 et 28 juin, 29 et 30 juin, 26 et 30 juin I972, soit 5-6, 3-4 et 7-8 jours avant le début de l'épiaison ( $5 \mathrm{p}$. Ioo d'épis sortis).

Ils ont été préparés avec addition d'acide formique à raison de 2,8, 3,8 et 4,2 litres par tonne de fourrage frais respectivement. Ces différences s'expliquent par le fait que l'acide formique a été incorporé au fourrage par un même appareil distributeur monté successivement sur les trois ensileuses et que les quantités incorporées ont varié en sens inverse du débit des ensileuses. Les ensilages ont été conservés en silos couloirs bétonnés sous hangar; dès la fin du remplissage de chaque silo, ils ont été couverts d'un film plastique et chargés de ballots de paille. 


\section{Schéma expérimental}

24 vaches laitières de race Frisonne et Montbéliarde (dont 6 en première lactation) ayant vêlé au début de l'hiver, et maintenues depuis la rentrée à l'étable (en moyenne 3 semaines avant le vêlage) sur un régime commun composé d'ensilage de prairie naturelle et d'aliments concentrés, ont été appariées 3 par 3 en moyenne 52 jours après leur vêlage, en tenant compte du stade de lactation, de la production de lait et du poids vif des animaux.

Après la mise en lots, chaque lot de 8 animaux a été affecté au hasard à l'un des 3 ensilages expérimentaux, qui ont été offerts à volonté (Io p. Ioo de refus par jour) sans foin. Les trois premières semaines ont été considérécs comme période pré-expérimentale permcttant d'ajuster l'apport d'aliment concentré. En effet, dès l'ouverture des silos nous avons mesuré la digestibilité des ensilages avec un lot de 5 moutons. Compte tenu des valeurs énergétiques (en UF) estimées et des quantités d'ensilage ingérées par les vaches à la fin de période pré-expérimentale, nous avons calculé que la ration de base apportait en moyenne, pour l'ensemble des animaux, l'énergie nette correspondant aux besoins d'entretien et de production de I I $\mathrm{kg}$ de lait. Toutes les vaches, quel que soit l'ensilage reçu, ont alors reçu un aliment concentré à raison de o, 38 UF par $\mathrm{kg}$ de lait à 4 p. Ioo produit au-dessus de I I $\mathrm{kg}$ par jour ; la quantité d'aliment concentré distribuée était prédéterminée pour toute la durée de l'expérience en prévoyant une diminution de la production laitière de $\mathrm{I}, 5 \mathrm{p}$. Ioo par semaine pour les vaches en première lactation et de 2,O p. Ioo pour les multipares. La période expérimentale proprement dite a alors commencé (les vaches étant en moyenne dans leur $73^{\mathrm{e}}$ jour de lactation) et a duré Io semaines. Elle a été suivie d'une période post-expérimentale de 3 semaines sur un même régime composé de foin et d'aliments concentrés.

L'aliment concentré (à base de céréales et de tourteaux d'arachide) utilisé durant la période expérimentale contenait $\mathrm{i} 70 \mathrm{~g}$ de matières azotées et $70 \mathrm{~g}$ de compléments minéraux par $\mathrm{kg}$ brut, ce qui couvrait largement los besoins en azote et minéraux des animaux, ceux-ci recevant par ailleurs I5o g par jour d'un mélange minéral à base de phosphate bicalcique, enrichi en oligoéléments.

\section{Mesures}

Les animaux étaient en stabulation entravée sans paille ; les quantités d'ensilage ingérées par chaque vache ont été mesurées pendant 4 jours consécutifs chaque semaine par pesée des quantités offertes et refusées (5 à ro p. Ioo des quantités offertes) et détermination de la teneur en matière sèche de l'ensilage offert lors de chacune des deux distributions journalières et des refus journaliers.

Les quantités de lait ont été pesées chaque jour, matin et soir. Le taux butyreux (méthode Gerber) a été déterminé 4 jours par semaine sur un échantillon moyen de la traite du matin et du soir et la teneur en matières azotées (méthode au noir amido II B) a été déterminée chaque semaine sur un échantillon moyen des 4 traites du mercredi et du jeudi.

Les animaux ont été pesés durant 2 jours consécutifs à la mise en lots, au début, au milieu et à la fin de la période expérimentale, ainsi que 3 semaines après la fin de la période expérimentale, les animaux ayant alors été remis sur un régime commun (foin de prairie naturelle).

Les ensilages ont été échantillonnés et analysés chaque mois selon les méthodes utilisées au laboratoire (DEMARquilly, I973) pour déterminer leur qualité de conservation (pH, teneurs en acides organiques, en azote ammoniacal...).

\section{RÉSULTATS}

\section{Composition chimique et digestibilité des ensilages}

Les 3 ensilages ont été très bien conservés (tabl. I). L'addition d'acide formique à une dose d'autant plus élevée que la longueur de hachage était grande a donc probablement annulé toute différence dans la qualité de conservation qui aurait pu être liée à la finesse de hachage. Notons que la machine à fléaux a introduit un peu de terre dans l'ensilage. Notons également que l'ensilage à brins moyens contient un peu plus d'acide acétique que les 2 autres. En outre, la digestibilité de cet ensilage à brins moyens est légèrement plus faible, ce qui résulte de sa récolte à un stade végétatif un peu plus tardif et peut être de l'hétérogénéité de la prairie. Contrairement à 
ce que nous avions observé dans des essais précédents (IULPHy et DhMarquilir, r973) les quantités ingérées par les moutons ont été peu différentes d'un ensilage à l'autre, sans doute à cause de la faible longueur des brins longs (ro cm).

\section{TABLEAU I}

Composition chimiqu, caractéristiqu's fermentaires, digestibilité et ingestibilité sur montons de's ensilages étudiés

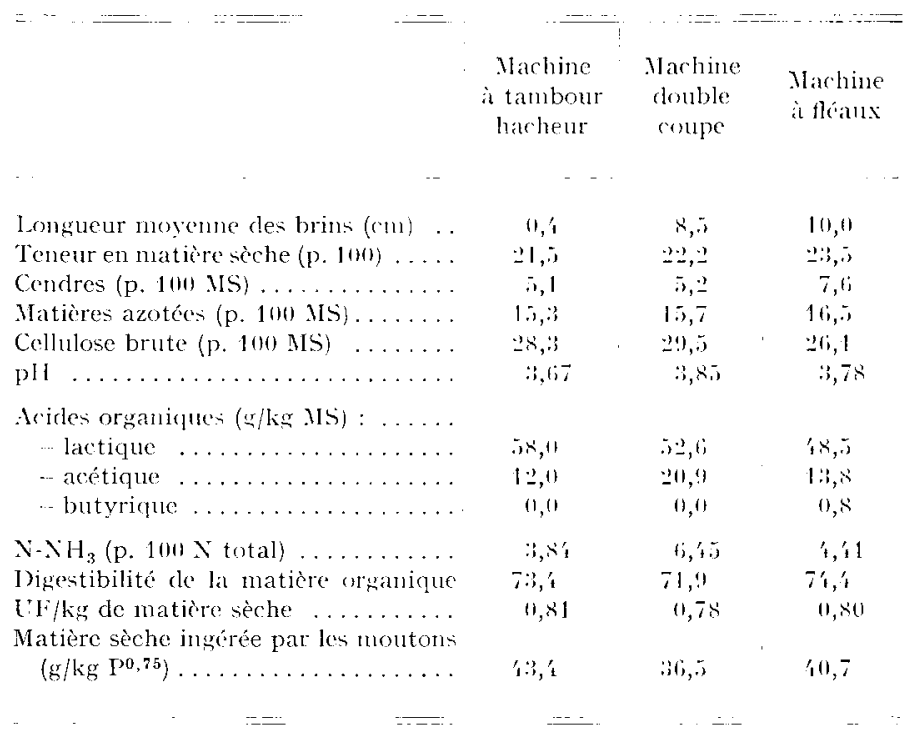

\section{Quantités ingérées}

Las trois ensilages ont été ingérés en quantités significativement $(\mathrm{P}<0, \mathrm{OI})$ différentes : $2,24, \mathrm{I}, 83$ et $2,00 \mathrm{~kg}$ de matière sèche par Io $\mathrm{kg}$ de poids vif respectivement pour les ensilages à brins courts, moyens et longs (tabl. 2). Avec 1'ensilage à brins courts, les vaches ont ingéré en plus par jour 2,37 kg de matière sèche d'ensilage (soit $+22,4$ p. I00) par rapport à l'ensilage à brins moyens et $\mathrm{I}, 53 \mathrm{~kg}$ de matière sèche (soit + - I3,2 p. IOo) par rapport à celui à brins longs. L'ensilage à brins dits "moyens" a été ingéré en quantité significativement $(\mathrm{P}<0, \mathrm{OI})$ plus faible que l'ensilage à brins "longs ». La plus faible ingestibilité de l'ensilage à brins moyens a dî être causée par sa plus faible digestibilité et sa plus forte taneur en acide acétique car la longueur de ses brins est très voisine de celle de l'ensilage à brins longs.

Les quantités de matière organique digestible d'ensilage ingérées ont été respectivement de $9,03,7,23$ et $7,86 \mathrm{~kg}$ soit une augmentation respective pour l'ensilage à brins courts de 24,9 et $\mathbf{1 4}, 9$ p. Ioo par rapport aux ensilages à brins moyens et longs. Les quantités d'énergie nette ingérées sous forme d'ensilage, en estimant la valeur énergétique nette des ensilages à partir de leur teneur en matière organique digestible (MOI) en $\mathrm{g}$ ) et indigestible (MONI) en $\mathrm{g}$ ) par la formule de BREIREM (I954) :

$$
\mathrm{UF} / \mathrm{kg} \mathrm{MS}=\frac{2,36 \mathrm{MOD}-\mathrm{I}, 20 \mathrm{MONI})}{\mathrm{I} 65^{\circ}}
$$




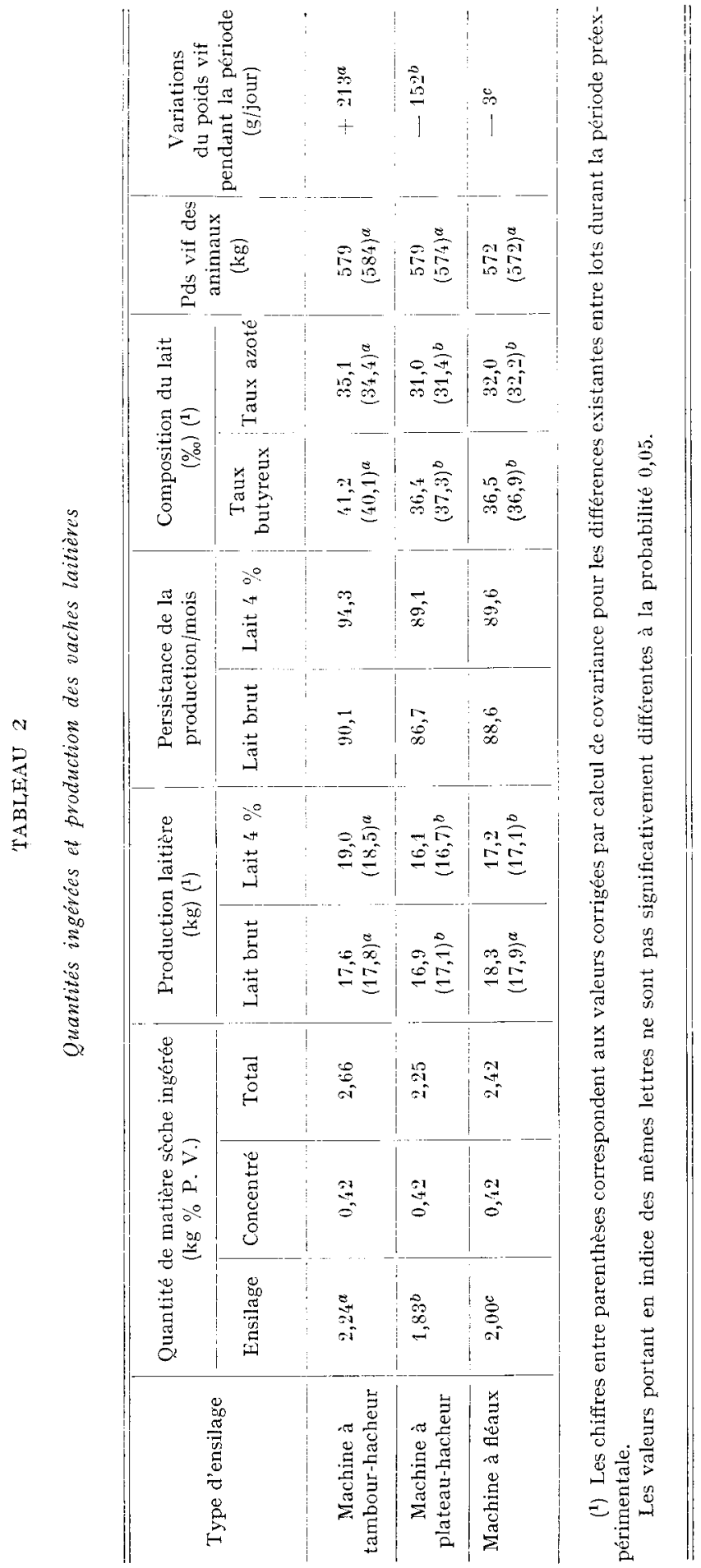


ont été respectivement de I0,5I, 8,27 et 9,I5 UF/jour/vache pour les ensilages à brins courts, moyens et longs. Les quantités correspondantes de matières azotées apparemment digestibles (MAD) ont été respectivement de I350, Iogo et I245 g/jour.

Comme cela était prévu, la quantité d'aliment concentré ingérée a été identique avec les trois ensilages. En estimant, d'après sa composition, la valeur nutritive de l'aliment concentré à $\mathrm{I}, \mathrm{I} 6 \mathrm{UF}$ et $\mathrm{I} 63 \mathrm{~g}$ de MAD par $\mathrm{kg}$ de matière sèche, la ration totale a apporté respectivement aux lots "brins courts ", "brins moyens " et "brins longs " I3,29, II,07 et II,97 UF/jour/vache pour l'énergie et I750, I490 et I650 g de MAD pour l'azote.

\section{Production laitière et composition du lait}

Les productions laitières, corrigées par analyse de covariance pour les différences existantes entre les lots durant la période pré-expérimentale, des lots ayant reçu les ensilages à brins courts ou à brins longs n'ont pas été significativement différentes entre elles mais elles ont été significativement $(\mathrm{P}<0,05)$ supérieures à celle du lot ayant reçu l'ensilage à brins moyens (tabl. 2). En revanche, la production de lait à 4 p. Ioo de matières grasses du lot " ensilage brins courts " a été significativement $(\mathrm{P}<0,05)$ supérieure à celle des "brins moyens " et " brins longs ", respectivement de I, 8 et I,4 kg de lait à 4 p. Ioo par jour et par vache, en raison du taux butyreux significativement plus élevé que celui des lots "brins moyens" $(P<0,05)$ et "brins longs" $(P<0,01)$. Le coefficient de persistance mensuelle du lait a, de même, été plus élevé pour le lot recevant l'ensilage à brins courts (90, I p. I0o) que ceux des lots recevant les ensilages à brins moyens et longs (respectivement 86,7 et 88,6 p. Ioo). D'ailleurs, lorsque les animaux ont reçu le même régime (foin + aliment concentré) lors de la période postexpérimentale, la production de lait à $4 \mathrm{p}$. Ioo a diminué beaucoup plus pour le lot "brins courts" ( $-2,8 \mathrm{~kg}$ en $2 \mathrm{I}$ jours) que pour les lots "brins moyens " et "brins longs" (respectivement - 0,7 et - I,3 kg), ce qui confirme l'effet nettement favorable sur la production laitière de l'ensilage à brins courts par comparaison aux 2 autres ensilages.

La teneur en matières azotées du lait a été également significativement $(\mathrm{P}<0,05)$ plus élevée pour le lot " brins courts ", probablement par suite du niveau plus élevé des apports énergétiques.

\section{Variation de poids vif des animaux}

Les variations de poids vif au cours des io semaines de la période expérimentale ont été significativement $(\mathrm{P}<0,05)$ différentes selon les lots: respectivement $+2 \mathrm{I} 3$, - I52 et - $3 \mathrm{~g} /$ jour pour les lots "brins courts " "brins moyens " "brins longs". Il est cependant possible que les différences entre lots aient été amplifiées par des différences dans les quantités de contenu digestif, bien que entre les 2 pesées les quantités ingérées totales de matière sèche aient peu varié (respectivement - I, I, - I,7 et - $0,6 \mathrm{~kg}$ de matière sèche par jour). 


\title{
Estimation de la valeur nutritive des ensilages
} $\grave{a}$ partir des performances des vaches

\author{
En prenant comme besoins énergétiques nets des vaches, I,5 $+\frac{\text { Poids vif (kg) }}{200} \mathrm{UF}$
} pour l'entretien, $0,38 \mathrm{UF} / \mathrm{kg}$ de lait et $\pm 4 \mathrm{UF}$ pour une variation en plus ou en moins d'un $\mathrm{kg}$ de poids vif, nous avons calculé la valeur énergétique nette " réelle " des ensilages à partir des performances réalisées par les vaches. Les valeurs trouvées sont respectivement de $0,74,0,67$ et $0,7 \mathrm{I} \mathrm{UP} / \mathrm{kg}$ de matière sèche pour les ensilages à brins " courts " " moyens " et " longs ". Elles sont inférieures à celles calculées à partir des digestibilités mesurées sur moutons $(0,8 \mathrm{I}, 0,78,0,8 \mathrm{o})$ et les différences entre ensilages sont nettement amplifiées. Compte tenu de ces valeurs " réelles 》 et des quantités d'ensilage ingérées, on peut calculer que ces trois ensilages couvraient en plus de l'entretien, les besoins énergétiques nets de production de $13,7,7$, I et 9,9 $\mathrm{kg}$ de lait 4 p. Ioo respectivement.

Il n'est pas possible de faire le même calcul pour les matières azotées digestibles (MAD) puisque dans les trois lots, les vaches ont reçu environ $300 \mathrm{~g}$ de MAD de plus que leurs besoins théoriques $(0,6 \mathrm{~g}$ par $\mathrm{kg}$ de poids vif pour l'entretien et $60 \mathrm{~g}$ par $\mathrm{kg}$ de lait 4 p. roo). En effet, les 3 ensilages couvraient théoriquement l'entretien + respectivement $\mathrm{I} 6,7, \mathrm{I} 2,4$ et $\mathrm{I} 5, \mathrm{I} \mathrm{kg}$ de lait et la ration totale respectivement l'entretien $+23,4$, I9, I et $21,8 \mathrm{~kg}$ de lait 4 p. IoO.

\section{DISCUSSION}

L'intérêt d'une machine de récolte hachant finement le fourrage ressort nettement de cet essai. L'ensilage à brins courts a été ingéré en plus grande quantité (en moyenne + I7,8 p. Ioo) que les ensilages à brins " moyens " et "longs " correspondants. Ces deux derniers ensilages différent malheureusement très peu dans la longueur réelle de leurs brins, l'ensileuse à double coupe (peut-être parce qu'elle était mal réglée) ne s'étant en effet guère montrée plus efficace pour le hachage que l'ensileuse à fléaux. L'augmentation d'ingestion entraînée par le hachage fin a été du même ordre que celle observée par Gordon et al. (I958), soit + II,4 p. Ioo et par Murdoch (I965), soit + I0,2 à $+20,9$ p. IOo. Elle aurait d'ailleurs été vraisemblablement encore plus importante si le hachage fin s'était accompagné d'une amélioration de la qualité de conservation, ce qui n'a pas été le cas dans cet essai, les doses d'acide formique ayant augmenté avec la longueur de hachage. Or, on sait que l'acide formique augmente la qualité de conservation des ensilages (SAUE et BREIREM, I969), et par là, la quantité d'ensilage ingérée par les vaches laitières (CASTLE et WATSON, I970 et I973).

Dans cet essai, l'augmentation d'ingestion entraînée par le hachage fin a été beaucoup plus importante que celle que nous avons enregistrée lors des essais sur génisses (DUl,PHy et DEMARQUILLy, I975). I1 est possible que les vaches en lactation, parce qu'elles ont des besoins beaucoup plus élevés et doivent consommer davantage, soient plus sensibles que les génisses à la finesse de hachage des ensilages, d'autant que ces dernières avaient déjà des croissances journalières assez élevées avec les ensi- 
lages à brins moyens ou longs. En outre, la longueur réelle des brins des ensilages distribués aux génisses n'a pas été mesurée et on ignore si les différences de longueur de brins entre ensilages étaient plus ou moins importantes que dans cet essai. Par ailleurs, les différences de quantités d'ensilage ingérées par les vaches dans cet essai entre les ensilages à brins dits " moyens " ou " longs " montrent qu'à longueur de brins pratiquement identique il peut y avoir des différences importantes de quantités ingérées suivant la qualité de conservation des ensilages.

Corrélativement à une ingestibilité plus élevée, les ensilages à brins courts ont aussi une valeur énergétique nette " réelle " plus élevée alors qu'ils ont le même coefficient de digestibilité de la matière organique que les ensilages à brins longs. Déjà nos essais sur génisses le laissaient soupçonner. Cette valeur plus élevée pourrait résulter d'une diminution du travail de mastication et de digestion mais aussi peutêtre d'une diminution de la digestion dans le rumen au bénéfice de la digestion dans l'intestin par suite d'une accélération du transit digestif.

L'ingestibilité et la valeur énergétique plus élevées des ensilages à brins courts ont entraîné une augmentation importante de la quantité d'énergie nette ingérée par les vaches (apport de 9,6 UF avec l'ensilage à brins courts contre 7,6 UF seulement avec les ensilages à brins longs) et par là une production laitière plus élevée, un meilleur coefficient de persistance de cette production, une augmentation de poids vif des animax et un taux azoté du lait plus élevé. On sait en effet que ce dernier varie dans le même sens que le niveau des apports énergétiques. En revanche, il est difficile d'expliquer pourquoi le hachage fin a aussi, dans cet essai, augmenté le taux butyretux du lait.

D'un point de vue zootechnique ces résultats montrent en outre que les ensilages d'herbe récoltés en coupe directe peuvent couvrir une partie importante des besoins énergétiques (entretien - I2 à I4 $\mathrm{kg}$ de lait) et azotés des vaches laitières dans la mesure où les fourrages sont récoltés au stade optimum (apparition des premiers épis pour les graminées), hachés finement et bien conservés.

Reçu pour publication en mars 1975 .

\author{
SUMMIARY
}

INFLUENCE, OF THE TYPE, OF FORAGE, HARVESTER

ON THE SILAGE INTAKE LEVEL AND THE PERFORMANCES OF DAIRY COWS

I. Dairy cows were used to compare three silages of fescue from the same field and harvested with three different machines : precision chop forage harvester (finely chopped silage : $0.4 \mathrm{~cm}$ ), double chop forage harvester (meanly chopped silage : $8.5 \mathrm{~cm}$ ), flail harvester (coarsely chopped silage : $10 \mathrm{~cm}$ ). Formic acid was added to all silages at harvesting and the preservation quality was good (table I).

2. The silages were offered ad libitum without hay to 3 comparable groups of 8 dairy cows. After a pre-experimental period of 3 weeks, the experiment was started on day 73 of lactation, on an average, and lasted ro weeks. Whatever the silage offered, the cows received a concentrate feed in the proportion of $0.3^{8} \mathrm{FU}$ per $\mathrm{kg} 4 \mathrm{p}$. roo fat-corrected milk produced over i $\mathrm{kg}$ per day. The amount of concentrate feed offered was predetermined for the whole experimental period, presupposing a $2 \mathrm{p}$. Ioo weekly reduction of the production of $4 \mathrm{p}$. Ioo fat-corrected milk.

3. The intakes of the 3 silages were significantly $(P<0.0 \mathrm{I})$ different: $2.24,1.83$ and $2.00 \mathrm{~kg}$ 
dry matter per $100 \mathrm{~kg}$ live weight respectively for finely, meanly and coarsely chopped silages (table 2).

4. Production of 4 p. I oo fat-corrected milk in the group receiving finely chopped silage was significantly $(\mathrm{P}<0.05)$ higher than that of the groups receiving meanly or coarsely chopped silages ( 18.5 versus 16.7 and $\mathrm{I} 7 . \mathrm{I} \mathrm{kg}$, respectively). This was mainly caused by the significantly higher butterfat level (40. I versus 37.3 and $36.3 \mathrm{~g} \mathrm{p}$. I ooo). Likewise, the crucle protein level of the milk was significantly higher for the group fed the finely chopped silage than for those fed the meanly and coarsely chopped ones (35.I versus 3 I.o and $32.0 \mathrm{~g}$ p. I 000).

5. The cows fed with the finely chopped silage exhibited a liveweight gain of $213 \mathrm{~g}$ per day during the experimental period of Io weeks whereas the cows receiving the meanly and coarsely chopped silages showed a liveweight loss of ${ }_{152}$ and $3 \mathrm{~g}$ per day respectively.

6 . On the basis of the silagc intake level and the performances of the dairy cows the "real " net energy values of the fincly, meanly and coarsely chopped silages were $0.74,0.67$ and $0.7 \mathrm{I} \mathrm{FU}$, respectively whereas the digestibilities of the organic matter measured on sheep were rather similar. Thus, on account of the amounts of silage ingested, these three silages satisfied not only the requirements for maintenance, but also the net energy requirements for production of 13.7 . 7. I and $9.9 \mathrm{~kg}$ respectively of 4 p. Ioo fat-corrected milk.

These results, showing that the voluntary intake and the energy valuc of finely chopped silages are higher than those of coarsely chopped silages, are discussed.

\section{RÉFÉRENCES BIBLIOGRAPHIQUES}

C.stre M. E., WATtion J. N., r97o a. Silage and milk production, a comparison between grass silages made with and without formic acid. J. Brit. Grassl. Soc., 25, 65-70.

Castle M. E., Watson J. N., r970 b. Silage and milk production, a comparison between wilted and unwilted grass silages made with and without formic acid. J. Brit. (irassl. Soc., 25, 278-284.

Castle M. E., Watson J. N., I973. Silage and milk production. A comparison between wilted grass silages made with and without formic acid. J. Brit. Soc., 28, 73-80.

DEMarquilly C., I973. Composition chimique, caractéristiques fermentaires, digestibilité et quantités ingérées des ensilages: moditication par rapport au fourrage vert initial. Ann. Zootech., 22, I-35.

Dulphy J.-P., Demarguiles C., ig73- Influence de la machine de récolte et de la finesse de hachage sur la valeur alimentaire des ensilayes. Ann. Zootech., 22, 199-217.

Dulphy J.-P., Demarquilix C., 1975. Influence de la machine de récolte sur la valeur des ensilages de graminées pour les génisses de race laitière. Ann. Zootech., 24, 35r-362.

Gordon C. H., Melin C. G., Wistiman H. G., IRwin H. M., McCalmont J. R., I9.58. Chemical quality, nutrient preservation, and fecding value of silages stored in bunker silos. J. Dairy Science, 41, I $738-1746$.

Murdocir J. C., 1965. The effect of length of silage on its voluntary intake by cattle. J. Brit. Grassl. Sioc., 20, 54-58.

Presthegge K., 1959. Experiments with grassland products for cattle. 93th Report of the Institute of Animal Nutrition. The Agricultural College of Norway.

Save O., Brerrem K., rg69. Formic acid as silage additive. Proc. 3rd Gen. meeting European crassl. Fed., 16I-172. 